\title{
Basic Study on Cognitive Conformity Behavior-Effects of Group Conditions on Behavior
}

\author{
Atsuo Murata \\ Okayama University, Okayama, Japan
}

\begin{abstract}
Cognitive conformity changes the opinion, the judgment, or the behavior of individuals and forces them to obey the norm or canon of the group or the organization. Such conformity might potentially lead to irrational decision-making as a group. Therefore, it is important for organizational managers to make efforts and take measures so that unconscious conformity behavior does not lead to serious accidents. An experiment was conducted to get insights into conformity behaviors that could potentially be risk factors of serious accidents. Put it more concretely, under a Prisoner's dilemma situation, it was experimentally explored how the number of members in a group and the situation of other member's decision-making, and whether the behavior is guided (derived) to cooperation or non-cooperation affected the conformity behavior. It tended that the conformity behavior to others frequently occurred when more than half of the members made the same decision, or when the decisions of other members was about to reach (gain) a majority. In short, the situation of other member's decision-making significantly $(p<0.01)$ affected the occurrence of conformity behavior. The guidance (derivation) to non-cooperation significantly $(p<0.01)$ led to more frequent conformity behaviors than the guidance (derivation) to cooperation. In other words, it was found that the conformity phenomena more easily occurred by the guidance (derivation) to non-cooperation than by the guidance (derivation) to cooperation.
\end{abstract}

Keywords: cognitive conformity, risk factor of accidents, decision-making, group condition

\section{Introduction}

In our society, it is a major issue to enhance cooperative behaviors (Axelrod, 1997, 2006; Komorita \& Parks, 1997). Without this, our society falls into social dilemma situations, and gets worse and worse. Such a situation in an organization leads to the violation of social or organizational rules, and at the worst case, it suffers from serious accidents or scandals. Cooperative and independent systems as a group have an advantage and lead to higher productivity (Rosenbaum, Moore, Cotton, Cook, Hieser, Shovar, \& Gray, 1980; Laughlin, VanderStoep, \& Hollingshead, 1991). On the other hand, the disadvantages and the negative aspects of group work are pointed out. Groupthink (Turner, Pratkanis, Probasco, \& Leve, 1993; Turner \& Pratkanis, 1998; Janis, 1982) is most likely to occur when a group experiences antecedent conditions such as high cohesion, insulation from experts, and limited methodological search and appraisal procedures, and to lead to symptoms such as illusion of invulnerability, belief in the inherent morality of the group, pressure on dissenters, self-censorship, and illusion of unanimity.

Atsuo Murata, Ph.D., professor, Department of Intelligent Mechanical Systems, Graduate School of Natural Science and Technology, Okayama University. 
As well as groupthink, cognitive obedience or conformity (Milgram, Bockman, \& Berkowitz, 1969; Milgram, 2009) also changes the opinion, the judgment, or the behavior of individuals and forces them to obey the norm or canon of the group or the organization. Similar behavior was also observed by Gerard, Williams, and Colloney (1968), and Latané and Wolf (1981). Deutsch and Gerard (1995) inferred the reason why we change our belief and obey to the majority as follows. Generally, we tend to be affected by the behavior of others especially under uncertain and ambiguous situations, and refer to the opinion or the behavior of others as a reference or a baseline of decision-making. This might be viewed as a heuristic response to available information. As stated in Kahneman (2011), this corresponds to availability bias (or bias that stem from availability heuristic), and distorts individual decision-making. On the basis of this, we must bear in mind that such conformity might potentially lead to irrational decision-making as a group.

Therefore, it is important for organizational managers to make efforts and take measures so that unconscious conformity behavior does not lead to serious accidents such as NASA (National Aeronautics and Space Administration) space shuttle challenger disaster occurred on January in 1986 (McConnell, 1987; Reason, 1990; Bredeson, 1999; McDonald, 2009; Engdahl, 2014), the root cause of which is regarded to be conformity behavior which forced each manufacturer related to the space shuttle to consent the launching of the space shuttle against the minor opinion that the shuttle might not be successfully launched (exploded) under severely cold weather.

The interactive effects of number of members in a group and the situation of other member's decision-making on conformity behavior have not fully explored. Here, the situation can be classified into: (1) when more than half of the members made the same decision; (2) when the decisions of other members was about to reach (gain) a majority; and (3) when no majority is reached or about to be reached. Moreover, it is not clear which of the two types of conformity, that is, the conformity to cooperation or the conformity to non-cooperation (defection) is easier to occur. This is of much interest and importance to obtain insights into conformity behavior. Therefore, it was hypothesized that the guidance (derivation) to non-cooperation significantly led to more frequent conformity behavior than the guidance (derivation) to cooperation under a Prisoner's dilemma situation. In other words, it was hypothesized that the conformity phenomena more easily occurred under the guidance (derivation) to non-cooperation than under the guidance (derivation) to cooperation.

The experiment was conducted to get insights into conformity behaviors that could potentially be risk factors of serious accidents. Put it more concretely, it was experimentally explored how the number of members in a group and the situation of other member's decision-making, and whether we are guided (derived) to cooperation or non-cooperation interactively affected the conformity behavior.

\section{Problems and Aim}

It is possible that cognitive conformity leads to a crucial accident. NASA space shuttle challenger disaster occurred on January in 1986. One of the root causes of this accident was analyzed as follows. Due to groupthink or conformity behavior, the manufacturer of O-ring was forced to consent the launching of the space shuttle in spite of recognizing that the shuttle might not be successfully launched (exploded) due to the malfunction of O-ring under severely cold weather.

Therefore, it is important to get insights into the prevention of accidents caused by conformity behavior. This study was conducted to get insights into conformity behavior that could potentially be risk factors of 
serious accidents. Put it more concretely, it was experimentally explored how the situation under which one faces decision-making affected the conformity behavior. Moreover, it was examined how the guidance to cooperation or non-cooperation affected the conformity behavior.

\section{Methods}

The experiment aimed at verifying the condition under which the conformity occurred in collective decision-making using a human $v s$. agent computer simulations under a Prisoner's dilemma situation.

\section{Participants}

Ten undergraduate or graduate students aged from 22 to 24 years took part in the experiment. They were all healthy and had no orthopedic disease. All signed the document on informed consent after receiving a brief explanation of the aim and the contents of the experiment.

\section{Task}

The experimental task was based on the repeated Prisoner's dilemma situation (Axelrod, 1997, 2006; Komorita, 1997) where the participants were required to carry out their own decision-making whether he or she selected cooperation or defection. This human $v s$. agent computer simulation was programmed on a personal computer. The participants were required to maximize their own interest in such a Prisoner's dilemma situation. The payoff matrix of the Prisoner's dilemma is summarized in Table 1. An example of display in the human vs. agent computer simulation is shown in Figure 1.

The number of repetition of the repeated Prisoner's dilemma game was set to 30 for each condition throughout the experiment.

\section{Design and Procedure}

The experimental factors were the number of members in a group, the situation of other member's decision-making, and the guidance condition (whether one is guided (derived) to cooperation or non-cooperation). All were within-subjects variables.

The number of members in a group included: single member, five members, and 15 members. Concerning the guidance (derivation) condition, the following two cases were used: (1) when intentionally conformed to cooperative behavior; and (2) when intentionally conformed to non-cooperative (defective) behavior. Each case included the following three situations (conditions): (1) when more than half of the members made the same decision; (2) when the decisions of other members was about to reach (gain) a majority; and (3) when no majority is reached or about to be reached. Experimental conditions are summarized in Table 2. For $n=5$, " $4(3,1), 1$ " means that more than half of the members (three members) have already made the same decision and one member has led to the different decision out of four members. The mark " $3(3,0), 2$ " means that three members have made the same decision and two members have not yet made decision. These correspond to (1) above mentioned. The mark " $3(2,1), 2$ " and " $2(2,0), 3$ " mean that the decisions of other members was about to reach (gain) a majority with the consent of one member. The mark " $1(1,0), 4$ " means that no majority is reached or about to be reached.

Here, the difference of the meaning between $n=15$ (proportion) and $n=15$ (meaning) is explained. As for $n=15, n=15$ (proportional) corresponds to merely three times of $n=5$. In short, the mark " $n=15$ (proportion), $9(6,3), 6$ " is merely three times of " $n=5,3(2,1), 2$ ", and has a different meaning from " $n=15$ (meaning), $9(7,2), 6$ ". Unlike " $n=5,3(2,1), 2$ ", the merely three times of " $n=5,3(2,1), 2$ " no longer represents the state 
under which the decisions of other members was about to reach (gain) a majority with the consent of one member. On the other hand, the mark " $n=15$ (meaning), 9(7,2),6", which was adjusted so that it represents the same meaning with " $n=5,3(2,1), 2$ ", clearly represents the same state with " $n=5,3(2,1), 2$ " where the decisions of other members was about to reach (gain) a majority with the consent of one member.

Table 1

Payoff Matrix of Prisoner's Dilemma Used in the Experiment

\begin{tabular}{llll}
\hline & & \multicolumn{2}{c}{ Opponent } \\
\cline { 3 - 4 } & & Cooperation & Defection \\
\hline \multirow{2}{*}{ Own } & Cooperation & $(500,500)$ & $(-1000,1500)$ \\
& Defection & $(1500,-1000)$ & $(-500,-500)$ \\
\hline
\end{tabular}

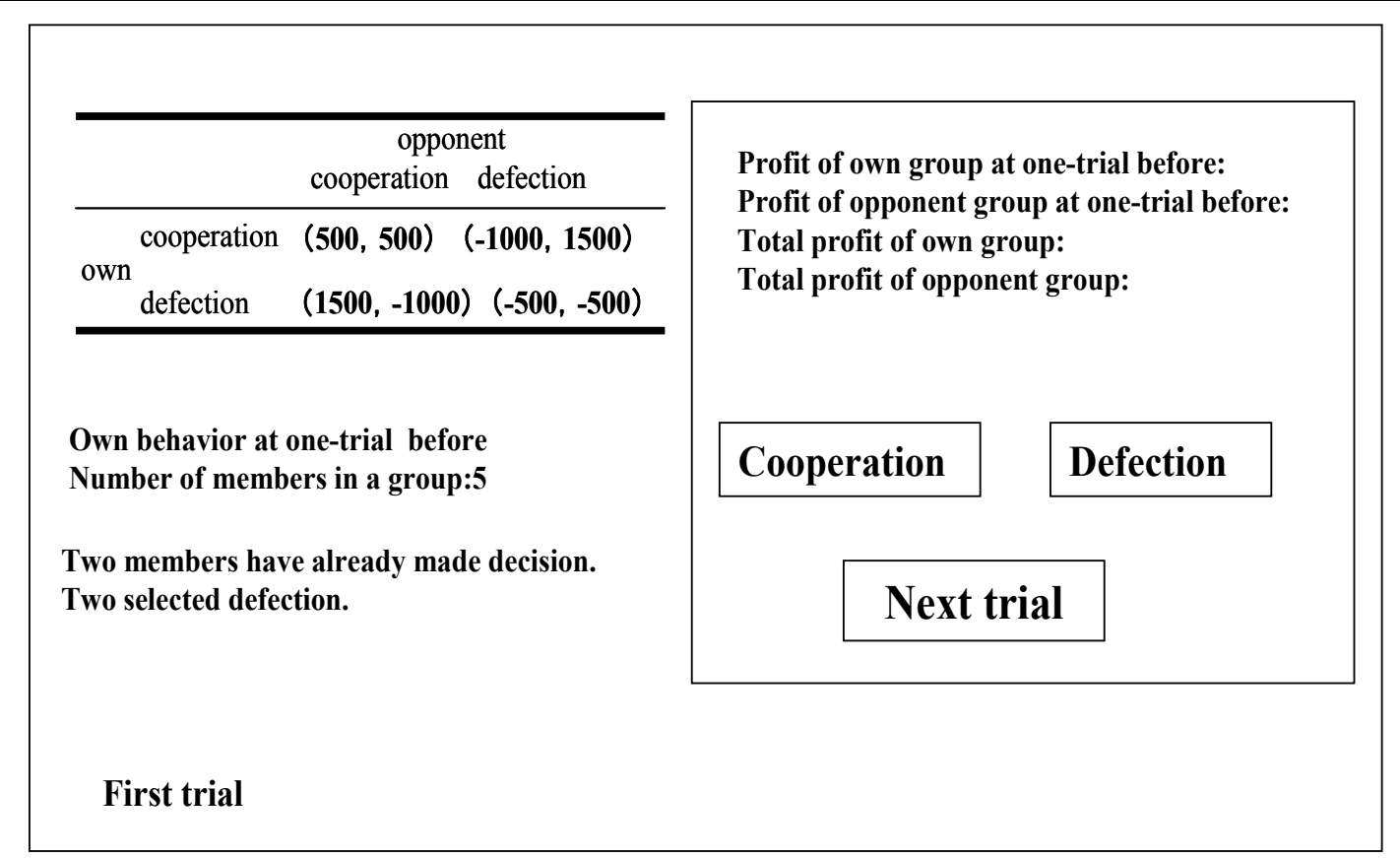

Figure 1. Examples of displays used in the experiment.

Table 2

Conditions of Number of Members in a Group and the Situation of Other Member's Decision-Making

\begin{tabular}{lrlrll}
\hline \multicolumn{5}{c}{} & \multicolumn{4}{c}{ Derivation (conformity) to cooperation } \\
\hline$n=5$ & $4(3,1) 1$ & $3(2,1) 2$ & $3(3,0) 2$ & $2(2,0) 3$ & $1(1,0) 4$ \\
$n=15$ (proportion) & $12(9,3) 3$ & $9(6,3) 6$ & $9(9,0) 6$ & $6(6,0) 9$ & $3(3,0) 12$ \\
$n=15$ (meaning) & $12(8,4) 3$ & $9(7,2) 6$ & $9(8,1) 6$ & $7(7,0) 8$ & $3(2,1) 12$ \\
\hline \multicolumn{5}{r}{} & \multicolumn{5}{c}{ Derivation (conformity) to defection (non-cooperation) } \\
\hline$n=5$ & $4(1,3) 1$ & $3(1,2) 2$ & $3(0,3) 2$ & $2(0,2) 3$ & $1(0,1) 4$ \\
$n=15$ (proportion) & $12(3,9) 3$ & $9(3,6) 3$ & $9(0,9) 6$ & $6(0,6) 9$ & $3(0,3) 12$ \\
$n=15$ (meaning) & $12(4,8) 3$ & $9(2,7) 6$ & $9(1,8) 6$ & $7(0,7) 8$ & $3(1,2) 12$ \\
\hline
\end{tabular}

A total of 15 conditions were included in both guidance (derivation) to cooperation and guidance (derivation) to defection. In the experiment, the participant repeated the experimental session (repeated Prisoner's dilemma game) three times for each condition in Table 2. The condition of conformity to cooperation or defection was counterbalanced across the participants. While half of participants firstly carried 
out the condition of deviation (conformity) to cooperation, half of participants firstly conducted the condition of derivation (guidance) to defection. A total of 45 experimental sessions were carried out for each conformity (derivation) condition. The order of 45 sessions was randomized across the participants.

The experimental conditions can be summarized as follows. The experimental factors included (1) the size of the group $(n=1,5,15)$; (2) the intentional guidance condition (intentionally guided (derived) to cooperation or non-cooperation); and (3) the situation of other member's decision-making (3-1: when more than half of the members made the same decision; 3-2: when the decisions of other members was about to reach (gain) a majority; 3-3: when no majority is reached or about to be reached). It was explored how such factors affected the conformity behavior.

\section{Results}

A one-way (group size) ANOVA carried out on the percentage cooperation revealed no significant main effect of group size. In Figure 2, the percentage cooperation is plotted as a function of number of members in a group. It tended that the percentage cooperation, as a whole, got higher with the increase of the number of members.

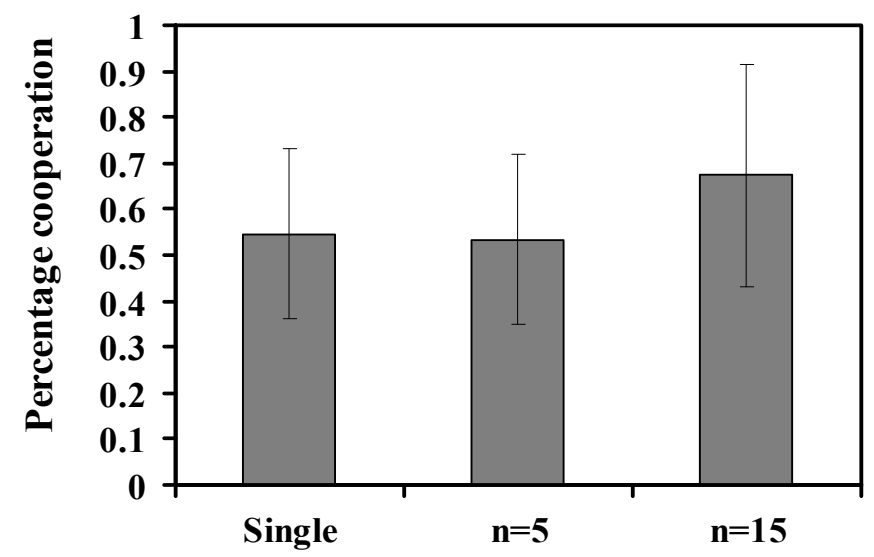

Figure 2. Cooperation rate as a function of number of members in a group.

A three-way (size of group by situation of other member's decision-making by guidance condition (whether the guidance was directed to cooperation or non-cooperation)) ANOVA carried out on the percentage cooperation revealed a main effect of situation of other member's decision-making $(F(2,18)=8.904, p<0.01)$. A size of group by situation of other member's decision-making interaction was also significant $(F(4,36)=$ 5.258, $p<0.01$ ). Fisher's PLSD (Protected Least Significant Difference) revealed a significant $(p<0.01)$ difference between 3-1 (when more than half of the members made the same decision) and 3-2 (when the decisions of other members was about to reach (gain) a majority with the consent of one member) conditions, and between 3-1 and 3-3 (when no majority is reached or about to be reached) conditions.

In Figure 3, the percentage cooperation is plotted as a function of the size of the group, and the situation of other member's decision-making when the participants were intentionally conformed to cooperative behavior. Figure 4 shows the percentage cooperation as a function of the size of the group and the situation of other member's decision-making when the participants were intentionally conformed to non-cooperative (defective) behavior.

A three-way (size of group by situation of other member's decision-making by guidance condition (whether the guidance was directed to cooperation or non-cooperation)) ANOVA carried out on the percentage 
conformity revealed a significant main effect of guidance condition $(F(1,9)=25.755, p<0.01)$. A guidance condition by situation of other member's decision-making interaction was significant $(F(2,18)=13.343, p<$ 0.01). A size of group by situation of other member's decision-making by guidance condition was also significant $(F(4,36)=4.884, p<0.01)$. Fisher's PLSD revealed a significant $(p<0.01)$ difference between the guidance to cooperation and the guidance to non-cooperation.

In Figure 5, the percentage conformity is plotted as a function of the size of the group $(n=1,5,15)$, and the situation of other member's decision-making (3-1, 3-2, and 3-3). This graph corresponds to the case where decision-making was intentionally oriented to cooperative behavior. The positive vertical value represents the percentage conformity to cooperation. The negative vertical value represents the percentage of non-conformity (conformity to non-cooperation). In Figure 6, a similar graph to Figure 5 is demonstrated for the case when decision-making was intentionally oriented to non-cooperative (defective) behavior. The positive vertical value represents the percentage conformity to non-cooperation (defection). The negative vertical value corresponds to the percentage conformity to cooperation.

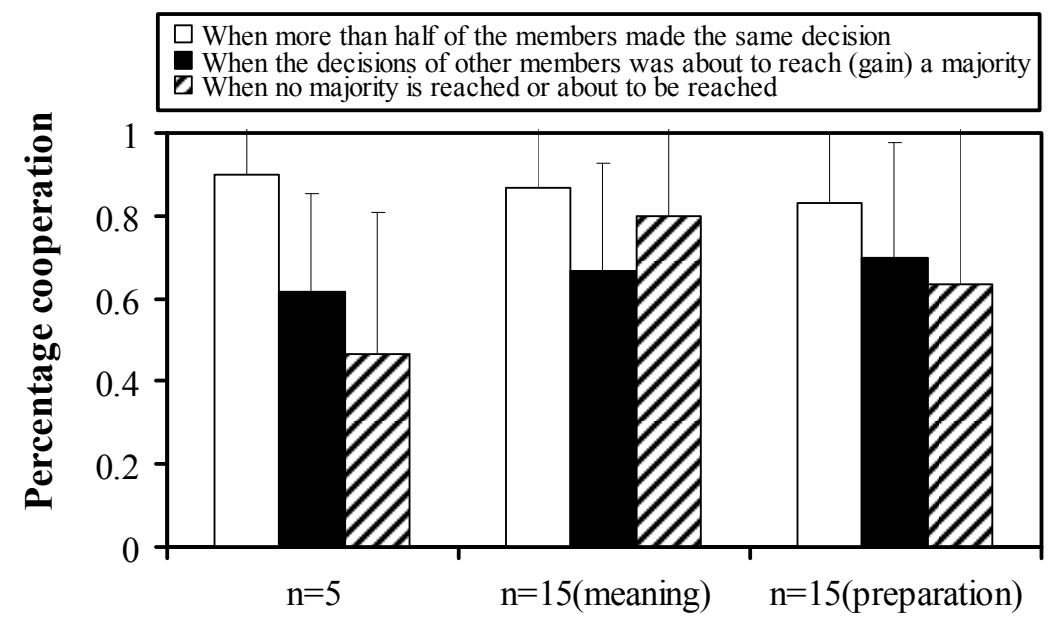

Figure 3. Percentage cooperation as a function of the size of the group, and the situation of other member's decision-making (when intentionally conformed to cooperative behavior).

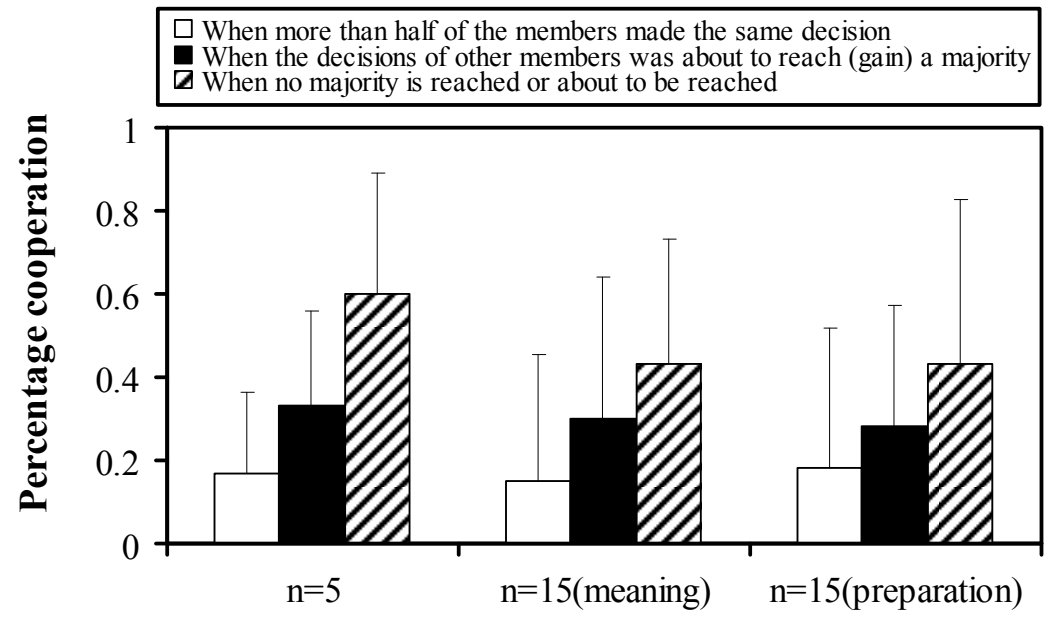

Figure 4. Percentage cooperation as a function of the size of the group, and the situation of other member's decision-making (when intentionally conformed to non-cooperative (defective) behavior). 


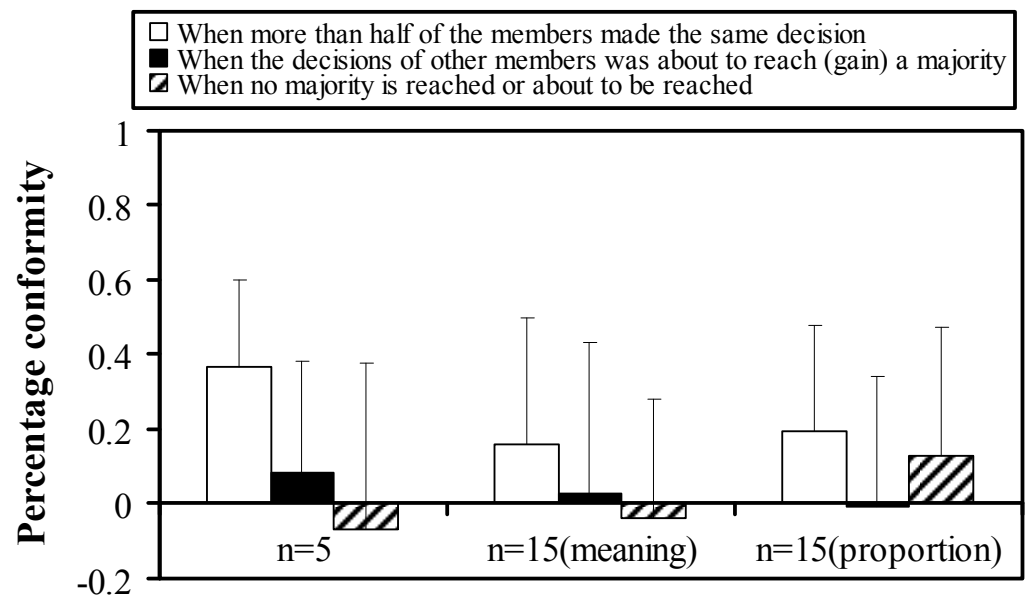

Figure 5. Percentage conformity as a function of the size of the group, and the situation of other member's decision-making (when intentionally conformed to cooperative behavior).

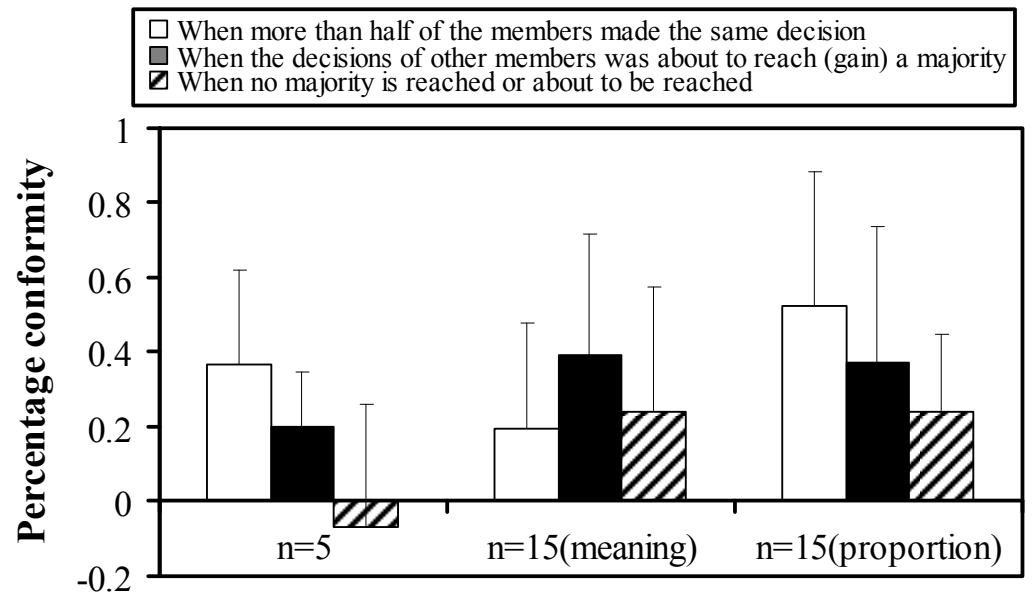

Figure 6. Percentage conformity as a function of the size of the group, and the situation of other member's decision-making (when intentionally conformed to non-cooperative (defective) behavior).

\section{Discussion}

The size of group did not affect the percentage correct (see Figure 2). This can be confirmed from the statistical analysis from Figure 3 to Figure 6. As shown in Figure 3, when the participants were intentionally induced to cooperative behavior, the percentage cooperation tended to be affected only by the situation of other member's decision-making (3-1: when more than half of the members made the same decision; 3-2: when the decisions of other members was about to reach (gain) a majority; 3-3: when no majority is reached or about to be reached). The significant size of group by situation of other member's decision-making interaction can be interpreted as follows. When no majority is reached or about to be reached, the increased number of members functioned to increase cooperation.

As shown in Figure 4, when the participants were intentionally induced to non-cooperative (defective) behavior, the percentage cooperation tended to be higher for $n=5$ than for $n=15$ especially when no majority is reached or about to be reached. Different from when the participants were intentionally induced to cooperative behavior (see Figure 3), it seems that the small number in the group $(n=5)$ might be helpful for the 
enhancement of cooperation. Moreover, the guidance condition (whether intentionally guided to cooperation or non-cooperation) was found to affect the percentage cooperation.

Although the conformity phenomenon was confirmed for both (1) when the participants were intentionally conformed to cooperative behavior; and (2) when the participants were intentionally conformed to non-cooperative (defective) behavior, the following different patterns of conformity were observed. When the participants were intentionally conformed to cooperative behavior, the fewer number of members $(n=5)$ in the group tended to induce conformity more frequently (see Figure 5). Contrary to this, the increased number of members in the group tended to lead to more frequent conformity when the participants were intentionally conformed to non-cooperative (defective) behavior (see Figure 6). The more percentage conformity when intentionally derived to non-cooperative (defective) behavior might be attributed to the nature of the experiment, in which the aim of the participant was the pursuit of own or organizational interest in a Prisoner's dilemma situation.

As hypothesized, the guidance (derivation) to non-cooperation significantly led to more frequent conformity behavior than the guidance (derivation) to cooperation. In other words, under a Prisoner's dilemma situation where the participant was required to maximize his/her own interest, the conformity phenomena more easily occurs under the guidance (derivation) to non-cooperation than under the guidance (derivation) to cooperation. This indicates that we must pay attention to the conformity behavior to defection especially when we are motivated to behave and maximize our own interest and prepare for the risks caused by such conformity behavior.

\section{Conclusions}

The aim of this study was to get insights into conformity behavior as a main cause of crucial accidents. The conclusions can be itemized as follows.

(1) It tended that the conformity behavior to others frequently occurred when more than half of the members made the same decision, or when the decisions of other members was about to reach (gain) a majority;

(2) Under the repeated Prisoner's dilemma situation, the guidance (derivation) to non-cooperation led to more frequently to the conformity behavior than the guidance (derivation) to cooperation. In other words, it was found that the conformity phenomena more easily occurred under the guidance (derivation) to non-cooperation than under the guidance (derivation) to cooperation.

Future research should confirm the validity of conformity phenomenon in more real-world settings. How such conformity leads to violation-based organizational error should be explored systematically in future research.

\section{References}

Axelrod, R. (1997). The complexity of cooperation. N.J.: Princeton, Princeton University Press.

Axelrod, R. (2006). The evolution of cooperation. M.A.:Cambridge, Basic Books.

Bredeson, C. (1999). The challenger disaster. N.J.: Berkley Heights, Enslow Publishers, Inc..

Deutsch, M., \& Gerard, H. B. (1995). A study on normative and informational social influence upon individual judgment. Journal of Personality and Social Psychology, 51, 629-636.

Engdahl, S. (2014). The challenger disaster. M.I.: Detroit, Greenhaven.

Gerard, H. B., Williams, R. A., \& Colloney, E. S. (1968). Conformity and group size. Journal of Personality and Social Psychology, 76, 129-142. 
Janis, I. L. (1982). Groupthink. M.A.: Boston, Wadsworth.

Kahmeman, D. (2011). Thinking, fast and slow. N.Y.: New York, Penguin Books.

Komorita, S. S., \& Parks, C. D. (1997). Social dilemmas. C.O.: Boulder, Westview Press.

Latané, B., \& Wolf, S. (1981). The social impact of majorities and minorities. Psychological Review, 88, 438-453.

Laughlin, P. R., VanderStoep, S. W., \& Hollingshead, B. (1991). Collective versus individual induction: Recognition of truth, rejection of error, and collective information processing. Journal of Personality and Social Psychology, 61(1), 50-67.

McConnell, M. (1987). Challenger - A major malfunction. N.Y.: New York, Doubleday \& Company, Inc..

McDonald, A. J. (2009). Truth, lies, and O-rings-Inside the space shuttle disaster. F.L.: Gainesville, University Press of Florida.

Milgram, S., Bockman, L., \& Berkowitz, L. (1969). Note on the drawing power of crowds of different size. Journal of Personality and Social Psychology, 13, 79-82.

Milgram, S. (2009). Obedience to authority. N.Y.: New York, Harper \& Raw Publishers.

Reason, J. (1990). Human error. N.Y.: New York, Cambridge University Press.

Rosenbaum, M. E., Moore, D. L., Cotton, J. L., Cook, M. S., Hieser, R. A., Shovar, M. N., \& Gray, M. J. (1980). Group productivity and process: Pure and mixed reward structures and task independence. Journal of Personality and Social Psychology, 39(4), 626-642.

Turner, M. E., Pratkanis, A. R., Probasco, P., \& Leve, C. (1993). Threat, cohesion, and group effectiveness: Testing a social identity maintenance perspective on group think. Journal of Personality and Social Psychology, 61(1), 50-67.

Turner, M. E., \& Pratkanis, A. R. (1998). Twenty-five years of groupthink theory and research: Lessons from the evaluation of a theory. Organizational Behavior and Human Decision Processes, 73(2/3), 105-115. 